\title{
Polarized nucleon-structure physics at RHIC-PHENIX
}

\author{
Yuji GOTO*i \\ (RIKEN Nishina Center for Accelerator-Based Science and RIKEN BNL Research Center) \\ E-mail: goto@bnl.gov
}

\begin{abstract}
For understanding the polarized structure of the nucleon, we have performed measurements with polarized proton collisions at RHIC employing the PHENIX detector. The spin of the nucleon is described as a sum of quark-spin contribution, gluon-spin contribution, and orbital motion of quarks and gluons inside the nucleon. We have investigated gluon-spin contribution with various reaction channels involving couplings to the gluons, and anti-quark contribution with weak boson production, both using longitudinally polarized proton collisions. These have resulted in a significant constraint on the helicity distribution of the gluon. Recent weak boson measurements promise a significant constraint, likewise, on the anti-quark helicity distributions. We have also studied transverse-spin phenomena, and will extend our study to measure 3-dimensional structure of the nucleon using transversely polarized proton collisions. We present recent results from PHENIX, and discuss our upgrade plans for the detector and the future measurements which they are aimed at.
\end{abstract}

XV International Conference on Hadron Spectroscopy

4-8/11/2013

Nara, Japan

\footnotetext{
* Speaker.

On behalf of the PHENIX collaboration.
} 


\section{Introduction}

How to understand the spin of the nucleon with an internal structure of the nucleon has been called the spin puzzle. A way to solve the puzzle is been established by recent progress of theoretical and experimental studies of the polarized nucleon structure. Experimentally, the polarized nucleon structure has been studied in high energy polarized lepton scattering experiments and hadron collision experiments based on the quark-gluon picture where the spin of the nucleon is described as a sum of polarization and orbital motion of partons, or quarks, anti-quarks and gluons, inside the nucleon.

RHIC at BNL is a unique polarized proton collider in the world where polarized proton collision experiments have been performed at $\sqrt{s}=62.4 \mathrm{GeV}$ to $510 \mathrm{GeV}$. As one of the large experiments at RHIC, the PHENIX experiment has performed helicity structure measurement of the nucleon with longitudinally polarized proton collisions and transverse structure measurement of the nucleon with transversely polarized proton collisions.

\section{Helicity structure of the nucleon}

The polarization of partons is measured as a helicity distribution inside the nucleon with longitudinally polarized proton collisions. We extract the polarization of partons by measuring double helicity asymmetries $A_{L L}=\left(d \sigma_{++}-d \sigma_{+-}\right) /\left(d \sigma_{++}+d \sigma_{+-}\right)$of various processes, where $d \sigma_{++}$ $\left(d \sigma_{+-}\right)$denotes the cross section of the reaction when two colliding particles have the same (opposite) helicity.

As the first goal of the experiment, we have studied the gluon polarization inside the proton, $\Delta g(x)$, by measuring $A_{L L}$ of inclusive neutral pions [1]. The neutral pions were detected by electromagnetic calorimeters with fine segmentation in the PHENIX central arms which cover midrapidity
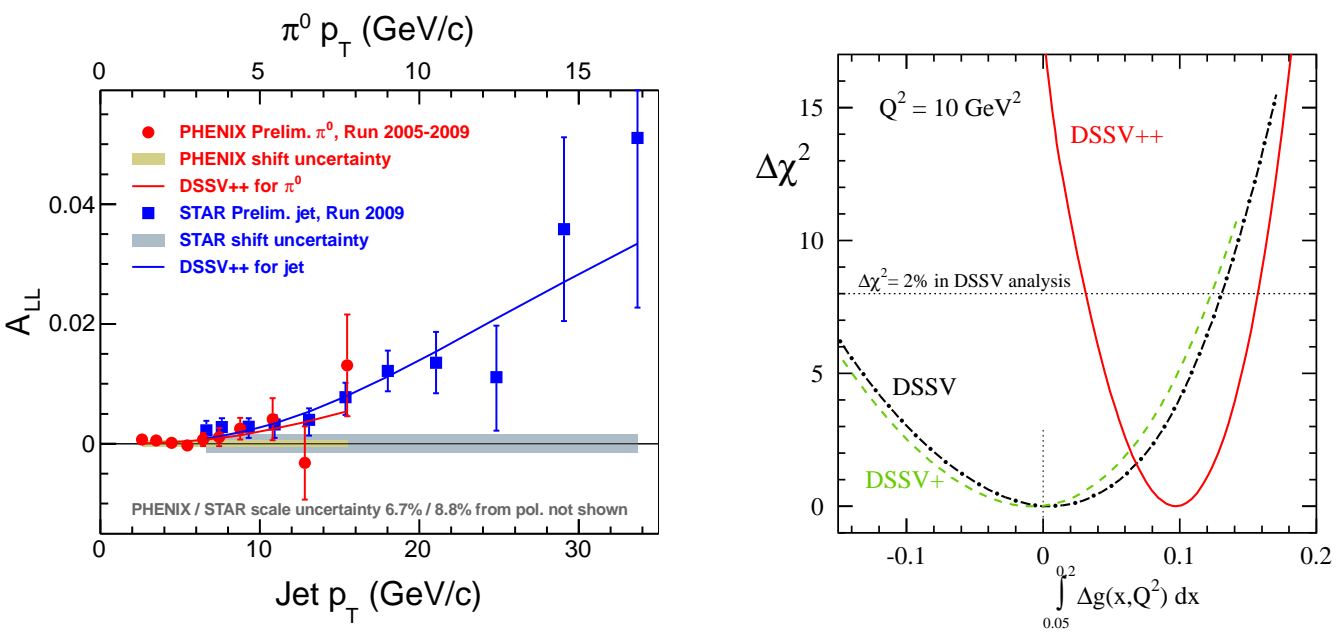

Figure 1: Left: $A_{L L}$ of $\pi^{0}$ measured by the PHENIX experiment and $A_{L L}$ of inclusive jet by the STAR experiment compared with the theory curve obtained by a QCD global analysis of the available experimental data. Right: $\chi^{2}$ profile of the integrated gluon contribution. 

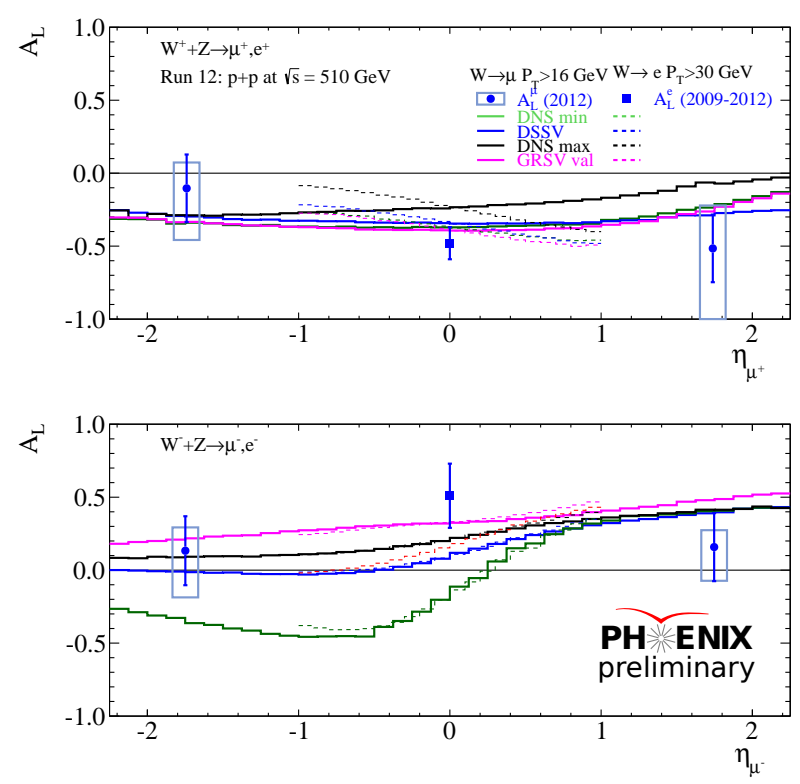

Figure 2: $A_{L}$ of $W^{+}$(upper panel) and $W^{-}$(lower panel) at central and forward/backward rapidities. Various theoretical predictions are shown together as dashed line for midrapidity and solid lines for forward/backward rapidities.

region, or pseudo-rapidity $|\eta|<0.35$. The left panel of Fig. 1 shows $A_{L L}$ of $\pi^{0}$ measured by the PHENIX experiment and $A_{L L}$ of inclusive jet by the STAR experiment [2] compared with the theory curve obtained by a QCD global analysis [3] of the available experimental data. The QCD global analysis shows the integrated gluon contribution $\int_{0.05}^{0.2} \Delta g(x) d x=0.1_{-0.07}^{+0.06}$ at a scale of $Q^{2}=$ $10 \mathrm{GeV}^{2}$. In the sensitive $x$ region $[0.05,0.2]$, the result indicate small positive gluon polarization. The right panel of Fig. 1 shows the $\chi^{2}$ profile of the integrated gluon contribution.

Another goal is determination of flavor-sorted helicity distribution of anti-quarks with weak boson measurements at $\sqrt{s}=500 \mathrm{GeV}$ and $510 \mathrm{GeV}$ [4]. Experimentally we measure parityviolating single-spin asymmetry $A_{L}$. From the chiral property of weak boson production and yield evaluation of decay leptons, the backward $A_{L}$ measurement of $W^{-}$to negative-lepton decay is sensitive to the $\bar{u}$-quark polarization, and the midrapidity measurement of $W^{+}$to positive-lepton decay is sensitive to the $\bar{d}$-quark polarization. Figure 2 shows 2012 result of the $A_{L}$ of weak bosons with the $W^{ \pm} \rightarrow e^{ \pm}$decay at the PHENIX central arms and $W^{ \pm} \rightarrow \mu^{ \pm}$decay at the PHENIX muon arms. In 2013, we have accumulated 5-times more statistics $\left(150 \mathrm{pb}^{-1}\right.$ luminosity and $52 \%$ polarization) of the longitudinally polarized proton collisions at $\sqrt{s}=510 \mathrm{GeV}$ for the final result of the asymmetry measurement of weak bosons.

\section{Transverse structure of the nucleon}

Transverse structure of the nucleon shows us nucleon structure beyond the simple parton picture. It shows many-body correlation of partons and presents the orbital motion inside the nucleon. It is described by extending or generalizing picture of the parton distribution, and measured with 


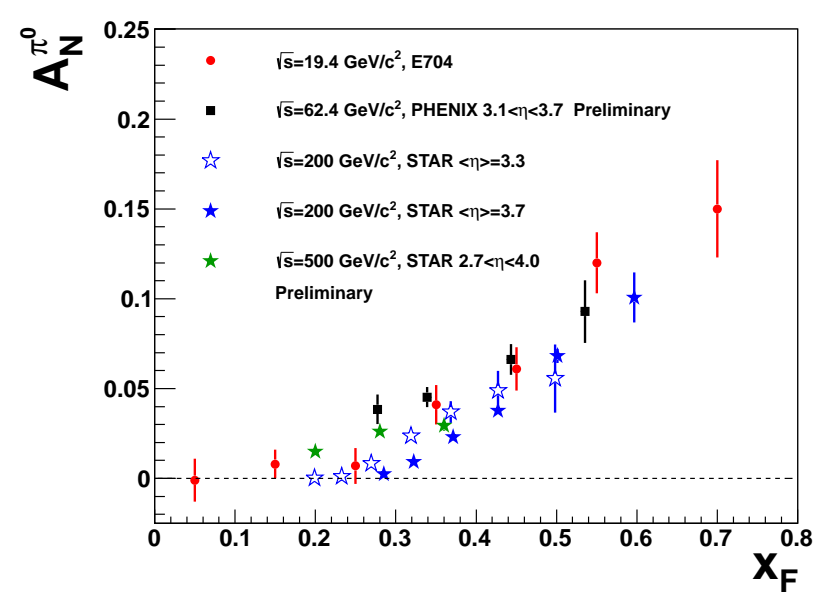

Figure 3: $A_{N}$ measurements for neutral pions at different center-of-mass energies.

transversely polarized proton collisions. Experimentally, it is measured as the single transversespin asymmetry (SSA), or $A_{N}=(d \sigma \uparrow-d \sigma \downarrow) /(d \sigma \uparrow+d \sigma \downarrow)$, where $d \sigma \uparrow(d \sigma \downarrow)$ is the production cross section when the protons are polarized up (down).

$A_{N}$ has shown unexpected large asymmetry in the forward-rapidity region and required development of many models base on perturbative QCD. Figure 3 shows measured $A_{N}$ from PHENIX [5] and other experiments. The large $A_{N}$ is described by several effects. One is so-called Sivers effect [6] with Sivers distribution function in the initial state which represents transverse-momentum dependence of partons inside the transversely-polarized nucleon. Another way is so-called Collins effect [7] with transversity distribution in the initial state combined with Collins fragmentation function in the final state. The transversity distribution shows correlation between transversely polarized nucleon and transversely polarized partons inside. The other way is the higher-twist effect which shows quark-gluon and multi-gluon correlation. It is important to distinguish these effects.

The preshower detector [8] installed in 2014 in front of the present forward electromagnetic calorimeter will enable us prompt photon asymmetry measurement which will distinguish Sivers effect and higher-twist effect, and Collins effect measurement with $\pi^{0}$ correlations with jet-like clusters.

\section{Future prospects}

The PHENIX experiment proposes substantial detector upgrades for long-term enhancement for major physics programs using full luminosity of recent upgraded RHIC accelerator [9]. Proposed midrapidity upgrades replaces the present magnet with a solenoid, and removes the large iron yoke at forward rapidity that provides the hadron absorber for the muon detectors. The open geometry of the forward direction of the midrapidity detector upgrades will allow for the addition of a forward spectrometer covering forward rapidity region, $1<\eta<4$ with capability of measuring hadrons, photons, electrons, muons and jets. We have been investigating requirements for detector 


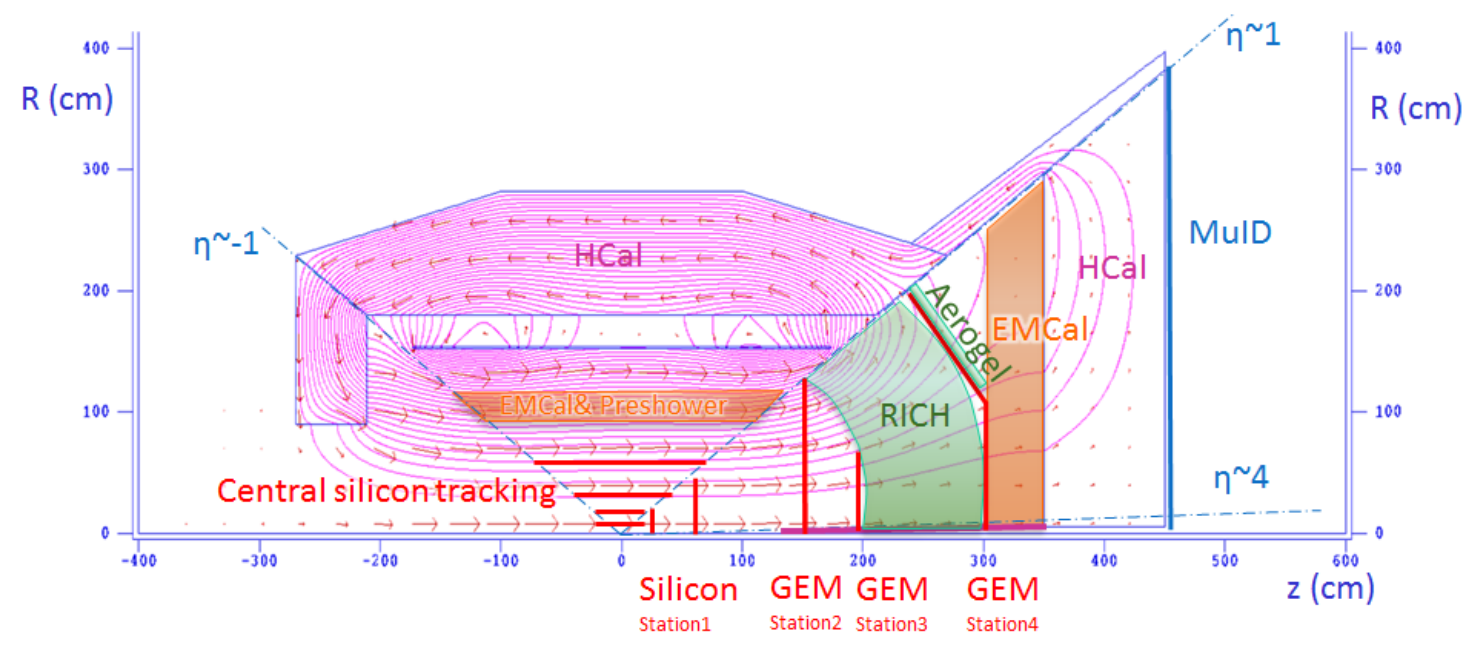

Figure 4: A cross section through the top-half of the upgraded detector concept. The magenta curves are contour lines of magnetic field potential.

design and performance of the forward upgrades consisting of charged-particle tracking (GEM), particle identification (RICH), electromagnetic and hadronic calorimeters as shown in Fig. 4.

The forward upgrades will extend our ability to investigate the Sivers and Collins effects at forward rapidities by performing full jet reconstruction; the Sivers effect through the observation of the SSA of final-state jet itself, and the Collins effect through an azimuthal anisotropy in the distribution of hadrons in jet. The asymmetry of single identified hadrons described by the Collins fragmentation function will give a measurement of quark transversity distribution at large $x$ which will determine the tensor charge of the nucleon and test Lattice QCD predictions for the tensor charge [10].

\section{References}

[1] A. Adare et al. [PHENIX Collaboration], arXiv:1402.6296 [hep-ex]; Phys. Rev. D 79, 012003 (2009); Phys. Rev. Lett. 103, 012003 (2009).

[2] L. Adamczyk et al. [STAR Collaboration], Phys. Rev. D 86, 032006 (2012).

[3] D. de Florian, R. Sassot, M. Stratmann and W. Vogelsang, Phys. Rev. D 80, 034030 (2009).

[4] S. Park [PHENIX Collaboration], PoS DIS 2013, 209 (2013); A. Adare et al. [PHENIX Collaboration], Phys. Rev. Lett. 106, 062001 (2011).

[5] A. Adare et al. [PHENIX Collaboration], arXiv:1312.1995 [hep-ex].

[6] D. W. Sivers, Phys. Rev. D 41, 83 (1990).

[7] J. C. Collins, Nucl. Phys. B 396, 161 (1993).

[8] S. Campbell et al. [PHENIX Collaboration], arXiv:1301.1096 [nucl-ex].

[9] C. Aidala et al.: arXiv:1207.6378 [nucl-ex].

[10] B. U. Musch, P. Hagler, J. W. Negele and A. Schafer, Phys. Rev. D 83, 094507 (2011). 